\title{
Nusinersen treatment of spinal muscular atrophy type 1 - results of expanded access programme in Poland
}

\author{
Sandra Modrzejewska ${ }^{1}$, Katarzyna Kotulska ${ }^{2}$, Ilona Kopyta ${ }^{3}$, Ewa Grędowska ${ }^{4}$, Ewa Emich-Widera ${ }^{3}$, \\ Katarzyna Tomaszek ${ }^{2}$, Justyna Paprocka ${ }^{3}$, Dariusz Chmielewski ${ }^{2}$, Jacek Pilch ${ }^{3}$, Jerzy Pietruszewski ${ }^{3}$, \\ Anna Lemska ${ }^{1}$, Marta Zawadzka ${ }^{1}$, Maria Mazurkiewicz-Bełdzińska ${ }^{1}$ \\ ${ }^{1}$ Department of Developmental Neurology, Medical University of Gdansk, Poland \\ ${ }^{2}$ Department of Neurology and Epileptology, The Children's Memorial Health Institute, Warsaw, Poland \\ ${ }^{3}$ Department of Paediatric Neurology, Medical University of Silesia, Katowice, Poland
}

${ }^{4}$ Biogen, Poland

\section{ABSTRACT}

Aim of the study. This study aimed to evaluate the effects of nusinersen therapy in Polish children with SMA type 1.

Clinical rationale of study. Spinal muscular atrophy (SMA) is a neuromuscular disorder that is characterised by the loss of motor neurons, progressive muscle weakness and atrophy, leading to increased disability and mortality. Nusinersen, an antisense oligonucleotide that promotes production of the functional survival motor neuron protein is approved for the treatment of SMA 5q in the European Union. In 2017, an early access programme (EAP) for nusinersen was launched in Poland. In this study, we present the results of nusinersen treatment in Polish patients participating in the EAP.

Materials and methods. We collected prospectively clinical data including mutational analysis of SMN1 and SMN2 genes, motor function outcomes as measured on a standardized scales, ventilatory and nutritional status, on SMA type 1 patients receiving nusinersen in three EAP centres in Poland. Scores on the CHOP-INTEND scale after 18-26 months of treatment were compared to baseline.

Results. We analysed data from 26 patients with SMA type 1, mean age 4.79 (2-15) years. The mutational analysis revealed two SMN2 gene copies in the majority of patients (61.54\%). Three and four copies were found in $34.62 \%$ and $3.84 \%$, respectively. Median disease duration was 21 months. Half $(n=13)$ of the patients required mechanical ventilation at baseline and $57.69 \%$ $(n=15)$ were fed by nasogastric tube or percutaneous endoscopic gastrostomy. No patient worsened during the follow-up. Mean improvement in CHOP-INTEND from baseline to the last follow-up was 7.38 points $(p<0.001)$. CHOP-INTEND scores did not decline for any patient. Patients with three or more SMN2 gene copies had higher scores than did the patients with two copies $(p=0.013)$, and they tended to show greater improvement over time, but the difference was not significant $(p=0.324)$. Shorter disease duration and higher CHOP-INTEND baseline score were associated with a better response $(p=0.015)$. Patients with a CHOP-INTEND score above the median had higher scores overall than the rest $(p<0.0013)$, and they improved significantly more than the rest $(p=0.037)$. Nusinersen was well tolerated, no new safety findings were identified.

Conclusions and clinical implications. Our data indicates that nusinersen treatment might be effective in SMA type 1 patients, regardless of their age and functional status.

Key words: nusinersen, spinal muscular atrophy, antisense oligonucleotide, expanded access programme (Neurol Neurochir Pol 2021; 55 (3): 289-294)

Address for correspondence: Sandra Modrzejewska, Department of Developmental Neurology Medical University of Gdansk, Gdansk, Poland; e-mail: sandra.modrzejewska@gmail.com 


\section{Introduction}

Spinal muscular atrophy (SMA), a rare genetic disease belonging to a group of neuromuscular disorders, is the leading mono genetic cause of mortality in infants. It is characterised by the progressive loss of motoneurons resulting in progressive muscle weakness and atrophy [1-4]. SMA results from a homozygous mutation or deletion in the SMN1 (survival motor neuron) gene located on the long arm of chromosome 5. In affected individuals, the production of functional survival motor neuron (SMN) protein, which is necessary for motor neuron function and survival, is severely impaired [4-7]. Nusinersen, an antisense oligonucleotide has been approved in the United States in the European Union and elsewhere, for the treatment of $5 \mathrm{q}$ spinal muscular atrophy. Nusinersen increases the production of the full-length SMN protein by modifying splicing of the pre-mRNA of the SMN2 gene, almost identical to SMN1, located on the same chromosome $[4,8,9]$. Spinal muscular atrophy incidences of 10.3 and 13.5/100,000 live births were recorded in Poland in 2005 and 2015, respectively [10, 11]. According to reports collected from 122 laboratories in 27 European countries, the median incidence was 11.9/100,000 [6.3-26.7/100,000, (1: 3,900-16,000)] of live births in 2011-2015. In 2015, the number of newly diagnosed patients with SMA in Poland was 54, and in total in 2011-2015 it was 240 [11]. Type 1 SMA (Werdnig-Hoffmann Disease) is the most prevalent and accounts for over $50 \%$ of all new cases. It is characterised by the occurrence of symptoms up to six months of age, as well as the patient's inability to sit unsupported. Patients usually do not survive beyond the age of two years [12-14]. Alternative classification for type $1 \mathrm{SMA}$ is also recognized and utilizes subtypes $1 \mathrm{~A}-1 \mathrm{C}$. The most severe subtype $1 \mathrm{~A}$ is associated with symptoms onset at birth or within 2 weeks of infancy, symptoms occurring later on, but before 3 months of age refer to subtype $1 \mathrm{~B}$ and thereafter, up to 6 months of age to subtype $1 \mathrm{C}$ [10-12]. Severe hypotonia as well as paradoxical breathing with a bell-shaped chest, characteristic for type 1, have been observed in studied patients [14]. Motor development is disturbed; patients not only do not acquire extra skills, but also their existing skills worsen by an average of 1.27 points on the CHOP INTEND (Children's Hospital of Philadelphia Infant Test of Neuromuscular Disorders) scale per year $[15,16]$. Before reimbursement, only a small subgroup of type 1 patients was treated in Poland within an expanded access programme. The reimbursement of nusinersen, granted on 1 January 2019, has provided wide access to treatment, including patients of any SMA type and functional status and those diagnosed presymptomatically. 518 patients were being treated in this drug programme as of 29 February 2020 [17] The aim of this study was to show the impact of nusinersen treatment received in an expanded access programme in type 1 SMA patients in a Polish population.

\section{Methods}

\section{Patients and procedures}

We conducted prospective, longitudinal collection of data from three centres that participated in the expanded access programme in Poland. Health Ministry approval of the programme was given in December 2016, with the first patients beginning treatment in February 2017. Patients in the EAP had been dosed until March 2019, after which they were moved to the reimbursed treatment programme. According to global EAP inclusion criteria, only patients with type 1 SMA, confirmed by genetic testing (biallelic deletion of SMN1 gene) were approved for the treatment [18]. Additionally, as agreed by sites, the number of SMN2 copies had to be confirmed as well. Lumbar puncture contradictions were considered as a treatment exclusion factor [19]. An interview with parents was conducted before patients were qualified, with an explanation of the regime of applied standard of care, and clear treatment expectations were set. The attending physician or team leader also collected informed consent from the parents or legal guardians. Prior to drug administration, assessments were made of the patient's motor function using the CHOP-INTEND scale, physical state, respiratory, orthopaedic and nutritional status. Laboratory tests, i.e. morphology, arterial blood gas measurement, coagulation, biochemistry, general urine test, general CSF and CSF culture test were also performed. Patients received $12 \mathrm{mg}$ nusinersen via intrathecal administration on days $1,15,30,60$ and thereafter every four months. Most procedures were done without general or local anaesthesia. If required, local anaesthesia such as EMLA cream was used. Prior to administration, 3-5 ml of cerebrospinal fluid was withdrawn as recommended. Administration of nusinersen followed after carrying out a CHOP-INTEND 16-item functional measurement, used to evaluate children's motor skills on a 0-64 point scale, plus ventilatory and feeding status assessments. These assessments were performed prior to the first dose, and thereafter before the sixth (month 10) and at each subsequent 4 month dose. Laboratory tests and physical condition assessments were performed at each visit. Patients were kept under observation, as per site guidance, with a minimum of 24 hours required as per any lumbar puncture procedure. The cohort of patients under investigation had treatments initiated at different timepoints, thus the available last follow-up differed with minimum observation prior to the eighth dose up to the tenth dose. All three SMA treatment centres obtained approval from their ethics committee to conduct the study.

\section{Statistical analysis}

Data was presented with descriptive statistics. Mixed effects models for repeated measures were used to study the effect of time on the CHOP INTEND score (baseline $v$. follow-up), with time as a fixed effect and intercept as a random 
Table 1. Cohort characteristics

\begin{tabular}{|c|c|}
\hline Variable & Statistic \\
\hline Number of patients & 26 \\
\hline Female, n (\%) & $13(50)$ \\
\hline \multicolumn{2}{|l|}{ Age, years } \\
\hline Mean \pm SD & $4.79 \pm 3.23$ \\
\hline Median (IQR) & $3.58(3-5)$ \\
\hline Min-Max & $2.00-15.00$ \\
\hline p for Shapiro-Wilk test ${ }^{*}$ & $<0.001$ \\
\hline \multicolumn{2}{|l|}{ Age at disease onset, months } \\
\hline Mean \pm SD & $2.00 \pm 1.72$ \\
\hline Median (IQR) & $2.00(1.00-3.00)$ \\
\hline Min-Max & $0-6.00$ \\
\hline p for Shapiro-Wilk test & 0.007 \\
\hline \multicolumn{2}{|l|}{ Age at diagnosis, months } \\
\hline Mean $\pm S D$ & $11.38 \pm 25.28$ \\
\hline Median (IQR) & $6.50(3.00-8.75)$ \\
\hline Min-Max & $1-134$ \\
\hline p for Shapiro-Wilk test & $<0.001$ \\
\hline \multicolumn{2}{|l|}{ Age at treatment start, months } \\
\hline Mean $\pm S D$ & $36.57 \pm 39.25$ \\
\hline Median (IQR) & $23.00(12.25-41.25)$ \\
\hline Min-Max & $3.00-165.00$ \\
\hline p for Shapiro-Wilk test & $<0.001$ \\
\hline \multicolumn{2}{|l|}{ SMN2 copies, n (\%) } \\
\hline 2 & $16(61.54)$ \\
\hline 3 & $9(34.62)$ \\
\hline 4 & $1(3.84)$ \\
\hline \multicolumn{2}{|l|}{ SMN subtype } \\
\hline $1 \mathrm{~A}$ & $1(3.84)$ \\
\hline 1B & $21(80.76)$ \\
\hline $1 C$ & $4(15.38)$ \\
\hline \multicolumn{2}{|l|}{ Body weight } \\
\hline Mean \pm SD & $16.27 \pm 9.30$ \\
\hline Median (IQR) & $13.55(11.00-16.57)$ \\
\hline Min-Max & $9.50-50.50$ \\
\hline p for Shapiro-Wilk test & $<0.001$ \\
\hline Body weight $<3$ centile, $n(\%)$ & $9(34.61)$ \\
\hline
\end{tabular}

*when $p<0.05$, the distribution of variable differs significantly from normal distribution, thus non-parametric statistics are preferred (like median and IQR rather than mean and SD); IQR - interquartile range; SD - standard deviation

effect (Model A). Additionally, the effect of time was analysed depending on the number of SMN2 copies ( $2 v s .3$ or more copies, Model B) and baseline CHOP INTEND score (below median $v s$. above median, Model C). Spearman rank correlation coefficient was used to study the relationship between baseline CHOP INTEND scores and its on-treatment change. $\mathrm{P}<0.05$ was considered significant. All analyses were completed in the R software (version 3.52).

\section{Results}

\section{Baseline characteristics}

The mean age of analysed patients was $4.79(2-15)$ years. The majority $61.54 \%$ (16) had two SMN2 copies, $34.62 \%$ (9) had three SMN2 copies, and 3.84\% (1) had four SMN2 copies. Mean CHOP-INTEND score at baseline was $19.11 \pm$ 14.28 points. Most, $80.76 \%$ (21), patients were subtype $1 \mathrm{~B}$, with symptoms onset before age three months, but not immediately after birth that was associated with subtype 1A. Subtype 1C patients experienced an onset of weakness after the neonatal period, but, unlike other subtypes of type 1 SMA, these children acquired good head control. Characteristics of the cohort are summarized in Table 1. Regarding ventilatory status, 13, $50 \%$, patients required tracheostomy and respirator support, five, $19.23 \%$, required non-invasive support, and eight, $30.76 \%$, did not require ventilatory support. Feeding support by NGT (nasogastric tube) or PEG (percutaneous endoscopic gastrostomy) was required in $15,57.69 \%$, patients. Data on the baseline functioning of patients is captured in Table 2 .

\section{Follow-up}

According to agreed monitoring timelines, effectiveness for this analysis was focused on data starting from the eight dose as a minimum up to ninth dose, based on the last follow-up visit. Data considers CHOP-INTEND assessment at the $18^{\text {th }}$ up to $26^{\text {th }}$ months of treatment duration, which is one of the longest observation period since nusinersen treatment has started in real world setting. Mean score in CHOP-INTEND at last follow-up was 26.50 \pm 18.04 , which is a mean improvement of 7.38 points from baseline ( $\mathrm{p}<0.001 ; 95 \%$ CI, 4.69-10.07). Respirator support with tracheostomy at last follow-up (as a result of an acute lung infection) was required by three more patients versus baseline, while two patients who were previously on 24 hour support had decreased their dependency to only nighttime ventilation or while on acute infection. Follow-up data on functioning are summarized in Table 2.

Patients with three or more SMN2 copies had higher CHOP INTEND scores (15.25, 95\% CI: 4.02-26.47, $\mathrm{p}=0.013$ ) and tended to improve in functional tests more than the rest of the patients, but this effect was non-significant $(2.78,95 \% \mathrm{CI}$ : $-2.63-8.20, p=0.324)$. The effect of time remained significant $(\mathrm{p}=0.015)$, patients with CHOP INTEND baseline score above median had higher scores $(\mathrm{p}<0.001)$ and improved significantly more than the rest of the patients $(5.53,95 \% \mathrm{CI}$ : $0.62-10.44, p=0.037)$. First improvements were observed, starting from the administration of the fifth dose. Some improvements in patient physical status were not captured by the scales, including longer endurance and easier conduction of the exercises reported by leading physiotherapists. Summary of the data is shown in Figure 1 and in Model A-C. None of the patients worsened, one (no. 25) did not change, and the rest improved. Similarly to Model C, patients with higher baseline 
Table 2. Baseline and follow-up data on functioning

$\begin{array}{lcc}\text { Variable } & \text { Baseline } & \text { Follow-up } \\ \text { CHOP INTEND } & & \\ \text { Mean } \pm \text { SD } & 19.11 \pm 14.28 & 26.50 \pm 18.04 \\ \text { Median (IQR) } & 17.50(6.00-32.5) & 25.50(12.25-40.50) \\ \text { Min-Max } & 0-48.00 & 1-56.00 \\ \text { p for Shapiro-Wilk test } & 0.097 \\ \text { Respiratory support, } n(\%) & & 0.084 \\ \text { Noninvasive } & 5(19.23) & 5(19.23) \\ \text { None } & 8(30.76) & 5(19.23) \\ \text { Ventilation }>16 \mathrm{~h} \text { per day, } \mathrm{n}(\%) & 13(50.00) & 11(42.30) \\ \text { Tracheostomy, } \mathrm{n}(\%) & 13(50.00) & 16(61.54) \\ \text { NGT or PEG, } \mathrm{n}(\%) & 15(57.69) & 13(50.00)\end{array}$

Follow-up was led for 18-26 months NGT-Nasogastric tube; PEG-percutaneous endoscopic gastrostomy

Model A

$\begin{array}{lccccc}\text { Effect } & \text { Estimate } & \text { Std. error } & \text { 95\% Cl } & \text { t } & \\ \text { Intercept } & 19.11 & 3.191 & 12.76-25.46 & 5.99 & <0.001 \\ \text { Time } & 7.38 & 1.35 & 4.69-10.07 & 5.47 & <0.001\end{array}$

Change in CHOP-INTEND score was significant ( $p<0.001$; an increase of 7.38 points from baseline, $95 \% \mathrm{Cl}, 4.69-10.07$ )

\section{Model B}

$\begin{array}{lccccc}\text { Effect } & \text { Estimate } & \text { Std. error } & \text { 95\% Cl } & \text { t } & 3.708 \\ \text { Intercept } & 13.250 & 3.574 & 6.29-20.20 & 3.670 \\ \text { Time } & 6.313 & 1.720 & 2.94-9.67 & 0.001 & 2.647 \\ \text { SMN2 copies }(\geq 3) & 15.250 & 5.762 & 4.02-26.47 & 0.013 \\ \text { Time by no. of SMN2 copies interaction } & 2.787 & 2.774 & -2.63-8.20 & 0.324\end{array}$

Effect of time was significant $(6.31,95 \% \mathrm{Cl}: 2.94-9.67, \mathrm{p}=0.001)$; patients with three or more SMN2 copies had higher CHOP-INTEND scores $(15.25,95 \% \mathrm{Cl}: 4.02-26.47, \mathrm{p}=0.013)$ and tended to improve more than rest of patients, but this effect was non-significant $(2.79,95 \% \mathrm{Cl}:-2.63-8.20, \mathrm{p}=0.324)$

\section{Model C}

\begin{tabular}{|c|c|c|c|c|c|}
\hline Effect & Estimate & Std. error & $95 \% \mathrm{Cl}$ & $\mathbf{t}$ & $\mathbf{p}$ \\
\hline Intercept & 6.769 & 2.313 & $2.28-11.24$ & 2.927 & 0.006 \\
\hline Time & 4.615 & 1.777 & $1.14-8.08$ & 2.598 & 0.015 \\
\hline Baseline score (above median) & 24.692 & 3.271 & $18.35-31.02$ & 7.549 & $<0.001$ \\
\hline Baseline score by time interaction & 5.538 & 2.512 & $0.62-10.44$ & 2.204 & 0.037 \\
\hline
\end{tabular}

Effect of time remained significant $(p=0.015)$; patients with CHOP INTEND baseline score above median had higher scores $(p<0.001)$ and improved significantly more than rest of patients $(5.54$, 95\%Cl: $0.62-10.44, p=0.037)$

CHOP INTEND scores improved more than those with lower baseline scores (rho $=0.41, \mathrm{p}=0.033$ ) as shown in Figure 2.

\section{Safety}

Through the study period, we did not observe any adverse events related to nusinersen itself. Drug administration was tolerated well, without any major complications. The adverse events observed in patients were: post-lumbar puncture syndrome in four patients (15.38\%), respiratory tract infection in four patients $(15.38 \%)$, increased liver enzymes after gastrointestinal infection in two patients (7.69\%), and unsealed puncture site with temporary CSF leakage in two patients (7.69\%). We evaluated the safety profile to be favourable, and this was similar to the latest findings from a broad review of the nusinersen clinical trial programme [20].

\section{Discussion}

We conducted a prospective, longitudinal observation of type 1 SMA patients treated within the expanded access programme for nusinersen. We analysed 26 patients over a minimum 18 month follow-up period. We observed improved motor function in 25 patients with stabilisation in one, which, compared to the natural history of the disease and an annual deterioration of minus 1.27 points, can be considered to be a positive treatment outcome [15]. 


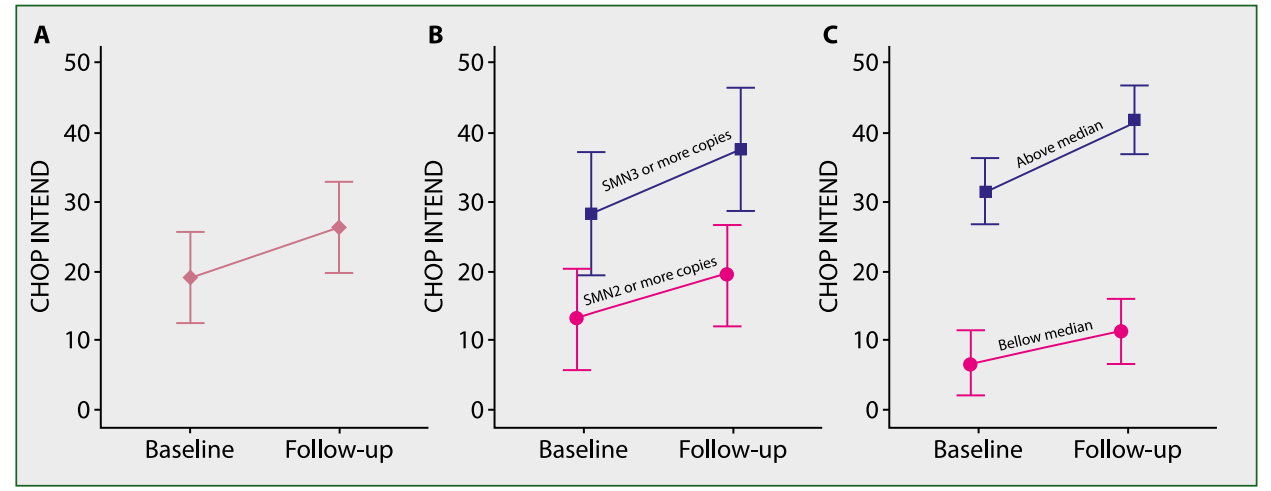

Figure 1. Change in CHOP INTEND score from baseline to follow-up among all patients (A) and depending on number of SMN2 copies (B) and baseline CHOP INTEND score (C). Plots show mean values and 95\% confidence intervals from linear mixed models for repeated measures with a random intercept and fixed effects of time (A, B, C), number of SMN2 copies (B), and baseline CHOP INTEND score (C)

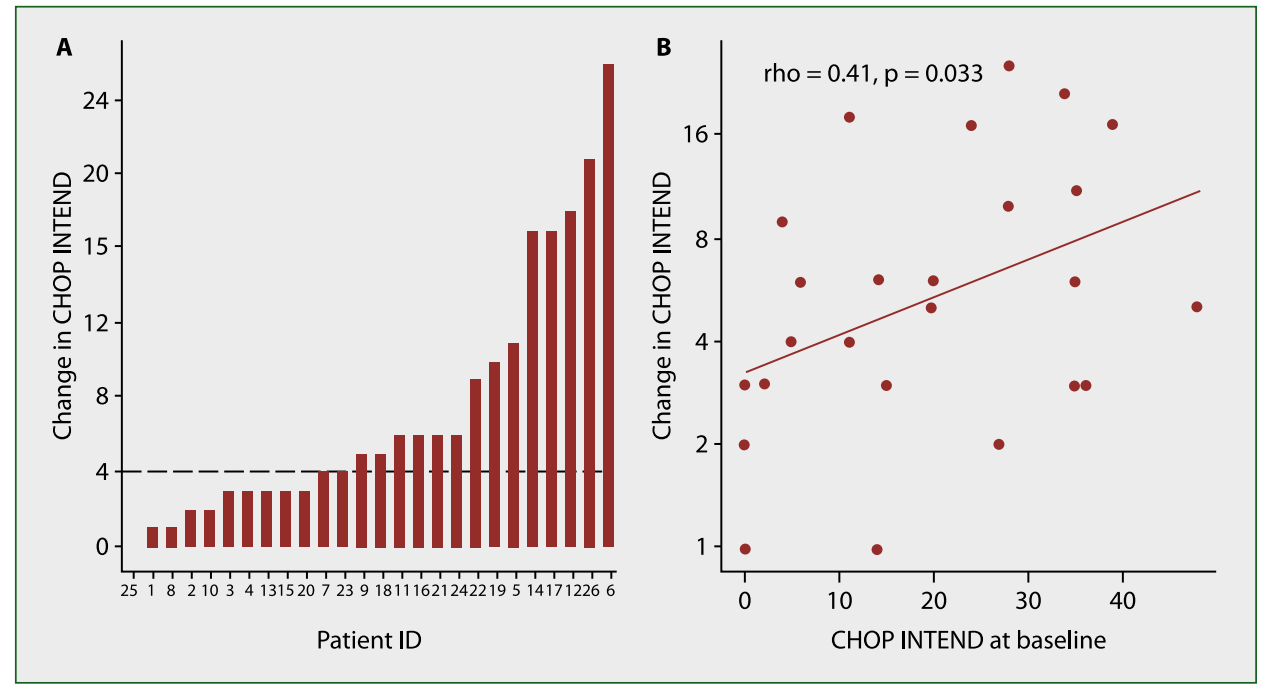

Figure 2. Change in CHOP INTEND score for individual patients (A) and correlation between baseline CHOP INTEND scores, with on-treatment change. No patient worsened, one (no. 25) did not change, and the rest improved. Similarly to Model C, patients with higher baseline scores improved more than those with lower baseline scores ( $r h o=0.41, p=0.033$ )

In our cohort, 16 patients (61.54\%) improved, measured by the clinically meaningful threshold of 4 or more points in CHOP-INTEND, with an individual change in one patient of up to 26 points. We acknowledge the potential gaps in the nusinersen clinical treatment programme, with very rigid criteria including patient ventilatory status and maximum age at screening [21]. The presented results can to some extent address those gaps. The cohort we analysed had more advanced stage of the disease, with $50 \%$ of the patients on tracheostomy and more than 16/24 hours use of a respirator, something which was an exclusion criterion in the phase III randomised, sham-control ENDEAR study [7]. Additionally, patients in our cohort had 21 months mean duration of the disease, whereas patients in ENDEAR at screening had a mean 13.2 weeks. Regarding body weight, nine (34.61\%) patients were also below the third percentile for age-adjusted body weight. We acknowledge remaining differences between the studies and limitations of our data related to the small group of patients, and differences in SMN2 copy number, study design or open label approach.

Nevertheless, we believe the results from the analysed cohort, together with the reports published by Pechman et al. and Farrar et al., can support a treatment rationale for type 1 patients, based on real-world data and clinical experience outside of the clinical trial programme $[22,23]$. In these two expanded access programme studies, improvement was seen in $77 \%$ and $100 \%$ respectively. Despite the baseline status of patients, we observed positive treatment effect regarding motor function in all. Our results also showed that patients with higher than median CHOP-INTEND scores at baseline and $3+$ SMN2 copies have generally better treatment outcomes, although this did not reach the point of statistical significance. We did not report major changes in patient respiratory status. 
Throughout the treatment period, three more patients underwent tracheostomy. At the same time in two patients we had decreased time needed on respirator support to nighttime ventilation and during the acute illness period.

\section{Clinical implications / future directions}

Our data shows that none of the analysed patients worsened, one stabilised, and the rest improved, despite the more advanced status of the disease, due to progressed ventilatory or feeding status. We found that patients with higher than median CHOP-INTEND scores at baseline and 3+ SMN2 copies presented better treatment responses.

Therefore, it is very important that patients suffering from spinal muscular atrophy receive treatment in the early stages of the disease. In Poland, this is now possible thanks to a national drug programme that provides treatment for all types of SMA patients. We hope to be able to treat patients with SMA before they develop clinical symptoms, which will be possible thanks to the implementation of newborn screening as standard.

Acknowledgements: Not applicable.

Conflict of interests: Ewa Grędowska is an employee of Biogen.

\section{References}

1. Wang $\mathrm{CH}, \mathrm{Xu} \mathrm{J}$, Carter TA, et al. Characterization of survival motor neuron (SMNT) gene deletions in asymptomatic carriers of spinal muscular atrophy. Hum Mol Genet. 1996; 5(3): 359-365, doi: 10.1093/ hmg/5.3.359, indexed in Pubmed: 8852661.

2. Zerres K, Rudnik-Schöneborn S, Forrest E, et al. A collaborative study on the natural history of childhood and juvenile onset proximal spinal muscular atrophy (type II and III SMA): 569 patients. Journal of the Neurological Sciences. 1997; 146(1): 67-72, doi: 10.1016/s0022$-510 \times(96) 00284-5$.

3. Frączek A, Potulska-Chromik A, Bednarska-Makaruk M, et al. Spinal muscular atrophy with an overlapping syndrome - „double trouble” or a potentially better outcome? Neurol Neurochir Pol. 2020; 54(5): 475-477, doi: 10.5603/PJNNS.a2020.0060, indexed in Pubmed: 32757203.

4. Jędrzejowska M, Kostera-Pruszczyk A. Spinal muscular atrophy - new therapies, new challenges. Neurol Neurochir Pol. 2020; 54(1): 8-13, doi: 10.5603/PJNNS.a2019.0068, indexed in Pubmed: 31922583.

5. Lorson $\mathrm{CL}$, Hahnen E, Androphy EJ, et al. A single nucleotide in the SMN gene regulates splicing and is responsible for spinal muscular atrophy. Proc Natl Acad Sci U S A. 1999; 96(11): 6307-6311, doi: 10.1073/pnas.96.11.6307, indexed in Pubmed: 10339583.

6. Monani UR, Lorson CL, Parsons DW, et al. A single nucleotide difference that alters splicing patterns distinguishes the SMA gene SMN1 from the copy gene SMN2. Hum Mol Genet. 1999; 8(7): 1177-1183, doi: $10.1093 / \mathrm{hmg} / 8.7 .1177$, indexed in Pubmed: 10369862.

7. Finkel RS, Mercuri E, Darras BT, et al. ENDEAR Study Group. Nusinersen versus Sham Control in Infantile-Onset Spinal Muscular Atrophy. N Engl J Med. 2017; 377(18): 1723-1732, doi: 10.1056/ NEJMoa1702752, indexed in Pubmed: 29091570.

8. Chiriboga CA, Swoboda KJ, Darras BT, et al. Results from a phase 1 study of nusinersen (ISIS-SMN(Rx)) in children with spinal mu- scular atrophy. Neurology. 2016; 86(10): 890-897, doi: 10.1212 WNL.0000000000002445, indexed in Pubmed: 26865511.

9. Gidaro T, Servais L. Nusinersen treatment of spinal muscular atrophy: current knowledge and existing gaps. Dev Med Child Neurol. 2019; 61(1): 19-24, doi: 10.1111/dmcn.14027, indexed in Pubmed: 30221755.

10. Jedrzejowska M, Milewski M, Zimowski J, et al. Incidence of spinal muscular atrophy in Poland--more frequent than predicted? Neuroepidemiology. 2010; 34(3): 152-157, doi: 10.1159/000275492, indexed in Pubmed: 20090376.

11. Verhaart IEC, Robertson A, Leary R, et al. A multi-source approach to determine SMA incidence and research ready population. J Neurol. 2017; 264(7): 1465-1473, doi: 10.1007/s00415-017-8549-1, indexed in Pubmed: 28634652.

12. Kolb SJ, Kissel JT. Spinal Muscular Atrophy. Neurol Clin. 2015; 33(4): 831-846, doi: 10.1016/j.ncl.2015.07.004, indexed in Pubmed: 26515624.

13. Wang $\mathrm{CH}$, Finkel RS, Bertini ES, et al. Participants of the International Conference on SMA Standard of Care. Consensus statement for standard of care in spinal muscular atrophy. J Child Neurol. 2007; 22(8): 1027-1049, doi: 10.1177/0883073807305788, indexed in Pubmed: 17761659.

14. D'Amico A, Mercuri E, Tiziano FD, et al. Spinal muscular atrophy. Orphanet J Rare Dis. 2011; 6: 71, doi: 10.1186/1750-1172-6-71, indexed in Pubmed: 22047105.

15. Finkel RS, McDermott MP, Kaufmann $\mathrm{P}$, et al. Observational study of spinal muscular atrophy type I and implications for clinical trials. Neurology. 2014; 83(9): 810-817, doi: 10.1212/ WNL.0000000000000741, indexed in Pubmed: 25080519.

16. De Sanctis R, Coratti G, Pasternak A, et al. Developmental milestones in type I spinal muscular atrophy. Neuromuscul Disord. 2016; 26(11): 754-759, doi: 10.1016/j.nmd.2016.10.002, indexed in Pubmed: 27769560.

17. Polish National Health Fund. 2020. Unpublished raw data.

18. Yong J, Moffett M, Lucas S. Implementing a Global Expanded Access Program (EAP) for Infantile-Onset Spinal Muscular Atrophy (Type I): Understanding the Imperative, Impact and Challenges. J Neuromuscul Dis. 2019; 6(2): 227-231, doi: 10.3233/JND-190387, indexed in Pubmed: 31127730.

19. Engelborghs $\mathrm{S}$, Niemantsverdriet $\mathrm{E}$, Struyfs $\mathrm{H}$, et al. Consensus guidelines for lumbar puncture in patients with neurological diseases. Alzheimer's \& Dementia: Diagnosis, Assessment \& Disease Monitoring. 2017; 8(1): 111-126, doi: 10.1016/j.dadm.2017.04.007.

20. Darras BT, Farrar MA, Mercuri E, et al. An Integrated Safety Analysis of Infants and Children with Symptomatic Spinal Muscular Atrophy (SMA) Treated with Nusinersen in Seven Clinical Trials. CNS Drugs. 2019; 33(9): 919-932, doi: 10.1007/s40263-019-00656-w, indexed in Pubmed: 31420846.

21. Meylemans A, De Bleecker J. Current evidence for treatment with nusinersen for spinal muscular atrophy: a systematic review. Acta Neurol Belg. 2019; 119(4): 523-533, doi: 10.1007/s13760-019-01199-z, indexed in Pubmed: 31388901.

22. Pechmann A, Langer T, Schorling D, et al. Evaluation of Children with SMA Type 1 Under Treatment with Nusinersen within the Expanded ACcess Program in Germany. J Neuromuscul Dis. 2018; 5(2): 135-143, doi: 10.3233/JND-180315, indexed in Pubmed: 29689734.

23. Farrar MA, Teoh HL, Carey KA, et al. Nusinersen for SMA: expanded access programme. J Neurol Neurosurg Psychiatry. 2018; 89(9): 937-942, doi: 10.1136/jnnp-2017-317412, indexed in Pubmed: 29549190 